\title{
A simplified model of jet power from active galactic nuclei
}

\author{
Y. Li, D.-X. Wang, and Z.-M. Gan
}

Department of Physics, Huazhong University of Science and Technology, Wuhan 430074, PR China

e-mail: dxwang@mail.hust.edu.cn

Received 9 January 2008 / Accepted 4 February 2008

\section{ABSTRACT}

\begin{abstract}
Aims. A simplified model of jet power from active galactic nuclei is proposed in which the relationship between jet power and disk luminosity is discussed by combining disk accretion with two mechanisms of extracting energy magnetically from a black hole accretion disk, i.e., the Blandford-Payne (BP) and the Blandford-Znajek (BZ) processes.

Methods. By including the BP process into the conservation laws of mass, angular momentum and energy, we derive the expressions of the BP power and disk luminosity, and the jet power is regarded as the sum of the BZ and BP powers.

Results. We find that the disk radiation flux and luminosity decrease because a fraction of the accretion energy is channelled into the outflow/jet in the BP process. It is found that the dominant cooling mode of the accretion disk is determined mainly by how the poloidal magnetic field decreases with the cylindrical radius of the jet. By using the parameter space we found, which consists of the black hole spin and the self-similar index of the configuration of the poloidal magnetic field frozen in the disk, we were able to compare the relative importance of the following quantities related to the jet production: (1) the BP power versus the disk luminosity, (2) the BP power versus the BZ power, and (3) the jet power versus the disk luminosity. In addition, we fit the jet power and broad-line region luminosity of 11 flat-spectrum radio quasars (FSRQs) and 17 steep-spectrum radio quasars (SSRQs) based on our model.
\end{abstract}

Key words. accretion, accretion disks

\section{Introduction}

Much attention has been paid to the relativistic jet and the enormous amounts of energy released in active galactic nuclei (AGNs) in the past decades. It is widely believed that the Blandford-Znajek (BZ) process (Blandford \& Znajek 1977, hereafter BZ77; Macdonald \& Thorne 1982) and the BlandfordPayne (BP) process (Blandford \& Payne 1982, hereafter BP82; Spruit 1996, hereafter S96) are the major mechanisms powering the relativistic jet from AGN hosting a supermassive black hole.

Energy and angular momentum are extracted from a rotating black hole to power the jet in the BZ process, in which the poloidal magnetic field lines connecting the black hole horizon with remote astrophysical loads are invoked. In the BP process, the disk matter is channelled into the outflow/jet by virtue of the poloidal magnetic field lines frozen in the disk, and the streaming gas is accelerated due to the work done by the magnetic torque. It has been argued that the kinetic flux carried by the outflow/jet driven centrifugally in the BP process always accompanies the Poynting flux (BP82; Camenzind 1986; S96).

Maraschi \& Tavecchio (2003, hereafter MT03) discuss the relation between the power carried by relativistic jets and the nuclear power provided by accretion for a group of blazars, including flat-spectrum radio quasars (FSRQs) and BL Lac objects. Their analysis indicates that the total jet power is of the same order of magnitude as the accretion power for FSRQs, while the jet luminosity is higher than the disk luminosity for BL Lac objects. The same result has been obtained by D'Elia et al. (2003).

Very recently, Liu et al. (2006, hereafter L06) investigated the relation between the jet power and the black hole mass in radio-loud AGNs. In their work, the jet power was estimated by using extrapolated, extended $151 \mathrm{MHz}$ flux density from the VLA $5 \mathrm{GHz}$ extended radio emission based on the formula derived by Punsly (2005), and the broad-line region luminosity and the black hole mass can be estimated by the broad emissionline luminosity (Celotti et al. 1997; McLure \& Dunlop 2001; McLure \& Jarvis 2002).

On the other hand, Miller et al. (2006) stress that disk accretion onto black holes is a fundamentally magnetic process: internal viscosity in some magnetic processes and disk winds can transfer angular momentum to drive disk accretion. It has been pointed out that an outflow emanating from an accretion disk can act as a sink for mass, angular momentum, and energy, altering the dissipation rates and effective temperatures across the disk (Donea \& Biermann 1996; Knigge 1999; Kuncic \& Bicknell 2007).

Motivated by the above works, we discuss the outflow/jet driven by the BP process, and investigate the interaction of the outflow/jet with the disk accretion based on the conservation laws of mass, angular momentum and energy. We find that the disk radiation flux and luminosity are reduced due to a fraction of accretion energy being channelled into the outflow/jet by the poloidal magnetic field frozen in the disk. It is shown that the dominant cooling mode in the disk is determined by the black hole spin $a_{*}$ and by the self-similar index $\alpha$ for the fixed-jet Lorentz factor. In addition, we find that the BP power is generally greater than the BZ power, except when the black hole spins very fast and the magnetic field decreases very steeply with the cylindrical radius. We compare the jet power and the disk luminosity and find that the jet power is almost the same as the disk luminosity. In this model the jet power is regarded as the sum of the BZ and BP powers, and the broad-line region luminosity is taken as a fraction of disk luminosity. In this way, 11 FSRQs and 17 steep-spectrum radio quasars (SSRQs) are fitted, and these results are consistent with those given in L06. 


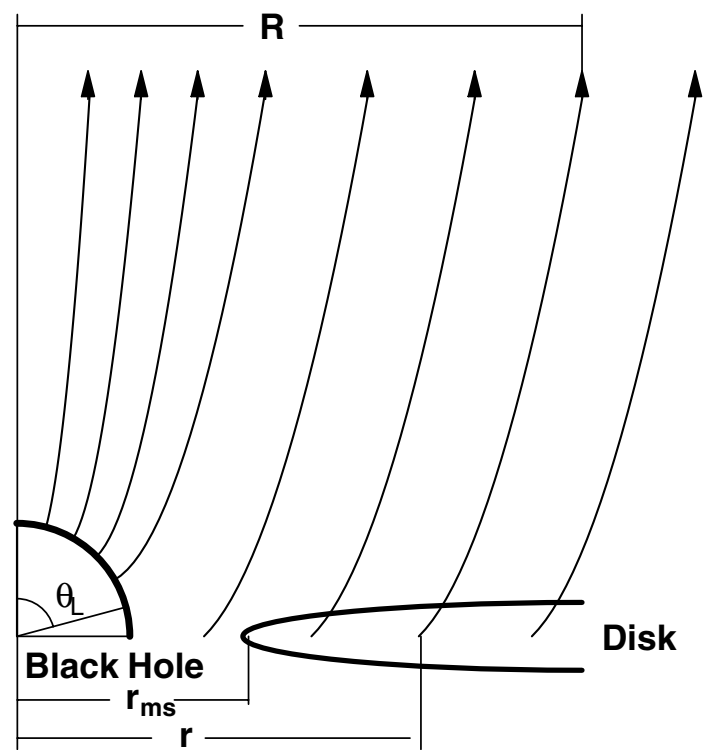

Fig. 1. Configuration of poloidal magnetic field threading a rotating black hole and its surrounding disk. We take $\theta_{\mathrm{L}}=0.5 \pi$ throughout this paper.

This paper is organized as follows. In Sect. 2 we describe our model and discuss the accretion rate and the radiation flux at the presence of a jet based on the conservation laws of mass, angular momentum, and energy. In Sect. 3 we compare the importance of the BP power to the disk luminosity, the BP power to the BZ power and the jet power to the disk luminosity in the parameter space consisting of the black hole spin and the self-similar index of the configuration of the poloidal magnetic field frozen in the disk. In addition, we fit the jet power and broad-line region luminosity of 11 FSRQs and 17 SSRQs. Finally, in Sect. 4, we summarize our main results and discuss the limitation of our model. Throughout this paper the units $G=c=1$ are used.

\section{Description of our model}

As is well known, large-scale magnetic fields anchored in the black hole accretion disk play essential roles in jet formation (Blandford 2002). Two scenarios have been proposed to interpret the origin of the large-scale magnetic fields. One is based on the results of some numerical simulations, indicating that smallscale magnetic fields could be amplified by virtue of a dynamo process in accretion disks (Hawley et al. 1995; Tout \& Pringle 1996; Amitage 1998). However, S96 thought that this would not be the ideal field for driving magnetic winds. Another possibility is that the magnetic field could be captured and advected inwards by the accreting matter in disks (BP82; Lovelace 1994; Spruit et al. 2005). The trapped large-scale fields can be strong enough to produce magnetic outflows.

In this paper we assume that the accretion disk is thin, Keplerian, stable, and perfectly conducting, located in an equatorial plane of a rotating black hole, and the inner edge of the disk is the last stable circular orbit (ISCO, Novikov \& Thorne 1973). The magnetic field configuration is assumed to be as shown in Fig. 1.

Following BP82, we assume that the poloidal magnetic field on the disk surface varies with the disk radius as

$B_{\mathrm{D}}^{\mathrm{p}}=B_{\mathrm{H}}^{\mathrm{p}}\left(r / r_{\mathrm{H}}\right)^{-5 / 4}$, where $r$ is the disk radius and $r_{\mathrm{H}}=M\left(1+\sqrt{1-a_{*}^{2}}\right)$ is the horizon radius of the black hole. The quantities $B_{\mathrm{D}}^{\mathrm{p}}$ and $B_{\mathrm{H}}^{\mathrm{p}}$ are the poloidal magnetic field at the disk surface and black hole horizon, respectively.

The poloidal magnetic field far from the disk surface is assumed to be roughly self-similar, being given as (BP82, Lubow et al. 1994)

$B^{\mathrm{p}} \sim B_{\mathrm{D}}^{\mathrm{p}}(R / r)^{-\alpha}, \quad(\alpha \geq 1)$

where $\alpha$ is the self-similar index to describe the variation of the poloidal magnetic field with the cylindrical radius $R$ of the jet.

Considering the balance between the magnetic pressure on the horizon and the ram pressure in the innermost parts of an accretion flow, Moderski et al. (1997) expressed the magnetic field at the horizon as

$\left(B_{\mathrm{H}}^{\mathrm{p}}\right)^{2} / 8 \pi=P_{\mathrm{ram}} \sim \rho c^{2} \sim \dot{M}_{\mathrm{acc}}\left(r_{\mathrm{ms}}\right) /\left(4 \pi r_{\mathrm{H}}^{2}\right)$,

where $\dot{M}_{\text {acc }}\left(r_{\mathrm{ms}}\right)$ is the accretion rate at ISCO with the radius $r_{\mathrm{ms}}$. Equation (3) can be rewritten as

$\dot{M}_{\mathrm{acc}}\left(r_{\mathrm{ms}}\right) \approx\left(B_{\mathrm{H}}^{\mathrm{p}}\right)^{2} r_{\mathrm{H}}^{2} / 2$.

The electromagnetic outflow/jet can be driven by the BZ process, in which the energy is extracted from a spinning black hole. The optimal BZ power is given (Wang et al. 2002)

$P_{\mathrm{BZ}} / P_{0}=2 A^{-1}\left(\arctan A-a_{*} / 2\right) /(1+q)^{2}$,

where $a_{*}$ is the black hole spin, and the quantities $P_{0}, A$, and $q$ are defined as $P_{0} \equiv \dot{M}_{\mathrm{acc}}\left(r_{\mathrm{ms}}\right) c^{2}, A \equiv \sqrt{(1-q) /(1+q)}$, and $q \equiv \sqrt{1-a_{*}^{2}}$, respectively.

As argued in BP82, the outflow matter could be accelerated centrifugally along the magnetic field lines, overcoming a barrier of gravitational potential to form magnetohydrodynamic (MHD) jets, provided that the poloidal magnetic field is strong and inclined enough. To avoid complexity in jet acceleration, Cao (2002, hereafter C02) expresses the mass loss rate in the jet from unit area of the disk surface as

$\dot{m}_{\mathrm{jet}}=\frac{\left(B_{\mathrm{D}}^{\mathrm{p}}\right)^{2}}{4 \pi} \frac{\left[r \Omega_{\mathrm{D}}\right]^{\alpha} \gamma_{\mathrm{j}}^{\alpha}}{\left(\gamma_{\mathrm{j}}^{2}-1\right)^{(1+\alpha) / 2}}$,

where $\gamma_{j}$ is the Lorentz factor of the jet. The quantity $\Omega_{D}$ is the Keplerian angular velocity at the foot point of the field line:

$\Omega_{\mathrm{D}}=\frac{1}{M\left(\xi^{3 / 2} \chi_{\mathrm{ms}}^{3}+a_{*}\right)}$,

where $\xi \equiv r / r_{\mathrm{ms}}$ is a radial parameter of the disk defined in terms of the radius $r_{\mathrm{ms}}$, and $\chi_{\mathrm{ms}}$ is defined as $\chi_{\mathrm{ms}} \equiv \sqrt{r_{\mathrm{ms}} / M}$.

According to the mass conservation law, the accretion rate of disk matter is related to the mass outflow rate by

$\mathrm{d} \dot{M}_{\mathrm{acc}}(r) / \mathrm{d} r=4 \pi r \dot{m}_{\mathrm{jet}}(r)$

where $\dot{M}_{\text {acc }}(r)$ and $\dot{m}_{\text {jet }}(r)$ are the accretion rate and the mass loss rate at foot point, respectively. Integrating Eq. (8), we have

$\dot{M}_{\mathrm{acc}}(r)=\dot{M}_{\mathrm{acc}}\left(r_{\mathrm{ms}}\right)+\int_{r_{\mathrm{ms}}}^{r} 4 \pi r^{\prime} \dot{m}_{\mathrm{jet}} \mathrm{d} r^{\prime}$,

where $\dot{M}_{\text {acc }}\left(r_{\mathrm{ms}}\right)$ is the accretion rate at ISCO. 


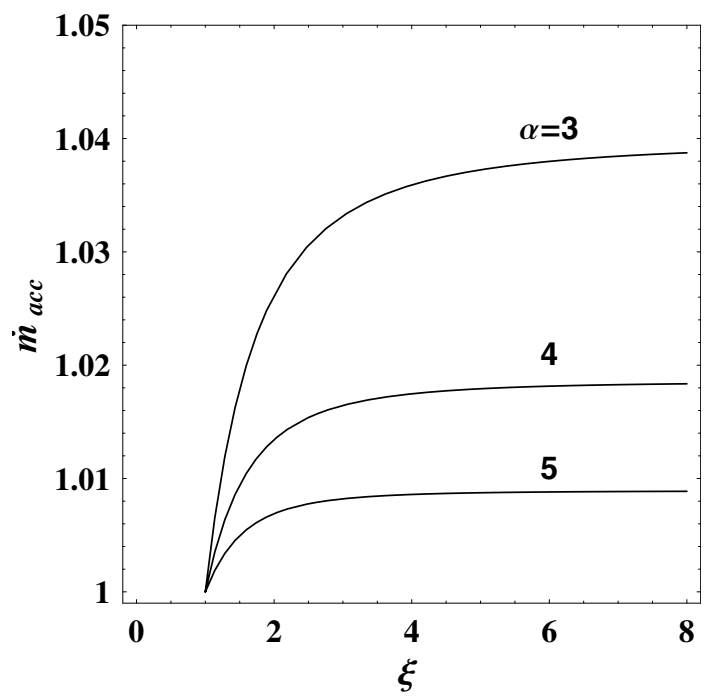

Fig. 2. The curves of $\dot{m}_{\text {acc }}$ versus $\xi$ for different values of $\alpha$ with $a_{*}=$ 0.95 and $\gamma_{\mathrm{j}}=5$.

Incorporating Eqs. (1), (4), (6), (7), and (9), we have the accretion rate

$$
\begin{aligned}
\dot{m}_{\mathrm{acc}}\left(a_{*}, \alpha, \gamma_{\mathrm{j}}, \xi\right) & =\dot{M}_{\mathrm{acc}}(r) / \dot{M}_{\mathrm{acc}}\left(r_{\mathrm{ms}}\right) \\
& =1+2 \int_{1}^{\xi} g_{\mathrm{jet}} \xi^{\alpha-3 / 2} \mathrm{~d} \xi^{\prime},
\end{aligned}
$$

where $g_{\text {jet }}$ is defined as

$g_{\text {jet }}=\left(r_{\mathrm{ms}} \Omega_{\mathrm{D}}\right)^{\alpha} \xi_{\mathrm{H}}^{1 / 2} \gamma_{\mathrm{j}}^{\alpha} /\left(\gamma_{\mathrm{j}}^{2}-1\right)^{(1+\alpha) / 2}$.

From Eq. (10) we find that the accretion rate at the given radius is determined by three parameters: the self-similar index $\alpha$, the Lorentz factor $\gamma_{\mathrm{j}}$, and the black hole spin $a_{*}$. Based on Eq. (10) we have the curves of the accretion rate $\dot{m}_{\text {acc }}$ varying with the radial parameter $\xi$ for the given values of $\alpha, a_{*}$, and $\gamma_{\mathrm{j}}$ as shown in Fig. 2. It is shown that $\dot{m}_{\text {acc }}$ increases very steeply with the increasing $\xi$ in the innermost region of the disk, while it almost stays constant because the disk radius is greater than several $r_{\mathrm{ms}}$. This result implies that the outflow is launched predominantly from the innermost region of the disk.

The ratio of the total mass-loss rate in the outflow to the accretion rate at ISCO is defined as

$\eta=\frac{\dot{M}_{\mathrm{acc}}\left(r_{\mathrm{out}}\right)-\dot{M}_{\mathrm{acc}}\left(r_{\mathrm{ms}}\right)}{\dot{M}_{\mathrm{acc}}\left(r_{\mathrm{ms}}\right)}=2 \int_{1}^{\xi_{\mathrm{out}}} g_{\mathrm{jet}} \xi^{\alpha-3 / 2} \mathrm{~d} \xi^{\prime}$,

where $\xi_{\text {out }} \equiv r_{\text {out }} / r_{\mathrm{ms}}$ is the dimensionless outer radius $r_{\text {out }}$ of the jet. For different values of $\alpha$, the curves of $\lg \eta$ versus $\gamma_{\mathrm{j}}$ with the given $a_{*}$, and those of $\lg \eta$ versus $a_{*}$ with the given $\gamma_{\mathrm{j}}$ are shown in Figs. $3 \mathrm{a}$ and $3 \mathrm{~b}$, respectively. It is shown in Fig. 3 that the ratio of the total mass-loss rate to the accretion rate decreases monotonically with increasing $\alpha$ and $\gamma_{\mathrm{j}}$, while it increases monotonically with increasing $a_{*}$. These results are consistent with those of $\mathrm{C} 02$.

Camenzind (1986) pointed out that the Poynting flux, as well as the kinetic flux, are carried by the winds driven centrifugally from the supermassive objects, and a fraction of the electromagnetic energy and angular momentum extracted is converted into the kinetic energy of matter in the outflow. It has been argued in BP82 and S96 that the Poynting flux dominates the kinetic flux near the disk surface, while the former is converted into the latter during accelerating matter in the outflow. Based on the calculations in BP82, the ratio of the Poynting flux to the kinetic flux is
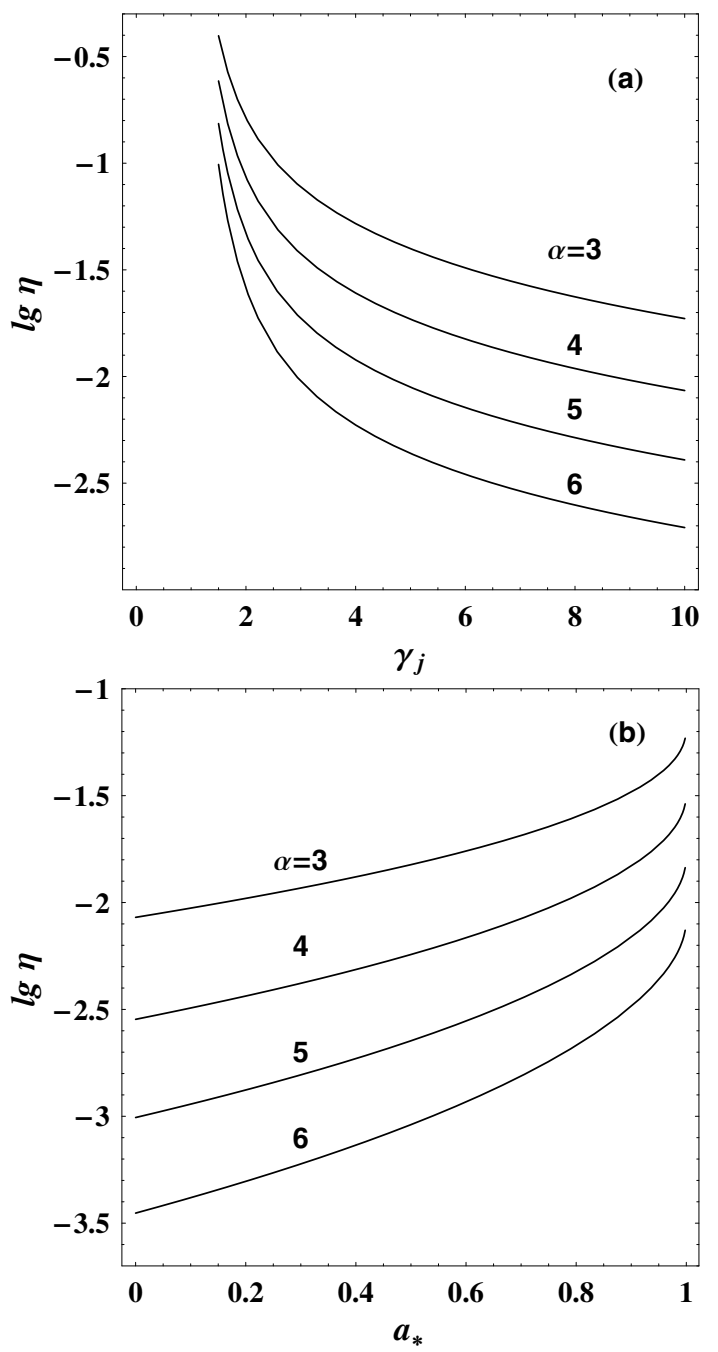

Fig. 3. The curves of $\lg \eta$ versus $\gamma_{\mathrm{j}}$ for $a_{*}=0.95$, b) the curves of $\lg \eta$ versus $a_{*}$ for $\gamma_{\mathrm{j}}=5$. In both cases $\alpha=3,4,5,6$ and $\xi_{\text {out }}=10^{4}$ are assumed.

about 58 near the disk surface, while it reduces to 2 at the Alfven surface. This result implies that about one third of the energy in the Poynting flux has been converted into the kinetic energy in the driving process.

Following $\mathrm{C} 02$, the kinetic flux of the jet can be written as

$F_{\text {jet }}=\dot{m}_{\text {jet }} c^{2}\left(\gamma_{\mathrm{j}}-1\right)$.

Considering that the Poynting flux is much greater than the kinetic flux near the disk surface, and about one third of the energy in the Poynting flux is converted into the kinetic energy of the jet, we can relate $F_{\text {jet }}$ at Alfven surface to the Poynting flux at the disk surface:

$S_{\mathrm{E}}=3 F_{\text {jet }}$.

According to BP82, the jet is driven from the disk surface due to the work done by the magnetic torques, and the angular momentum flux $S_{\mathrm{L}}$ extracted electromagnetically from the disk surface is related to the Poynting energy flux as

$S_{\mathrm{L}}=S_{\mathrm{E}} / \Omega_{\mathrm{D}}$.

Incorporating Eqs. (12)-(14), we have

$S_{\mathrm{L}}=3 \dot{m}_{\mathrm{jet}}\left(\gamma_{\mathrm{j}}-1\right) / \Omega_{\mathrm{D}}$. 
The integrated shear stress of the disk should be unavoidably affected by the transport of angular momentum and energy in the jet, resulting in the decrease of the disk dissipation and disk radiation. Unfortunately, this fact is neglected in $\mathrm{C} 02$, in which the strength of the large-scale field threading the disk is estimated based on dynamo mechanisms in the accretion disk and the expression of the integrated shear stress given by Novikov \& Thorne (1973).

At the appearance of the jet, the conservation equations of energy and angular momentum can be written as

$$
\begin{aligned}
& \frac{\mathrm{d}}{\mathrm{d} r}\left(\dot{M}_{\mathrm{acc}} E^{\dagger}-T_{\mathrm{visc}} \Omega_{\mathrm{D}}\right)=4 \pi r\left[\left(\dot{m}_{\mathrm{jet}}+F_{\mathrm{rad}}\right) E^{\dagger}+S_{\mathrm{L}} \Omega_{\mathrm{D}}\right], \\
& \frac{\mathrm{d}}{\mathrm{d} r}\left(\dot{M}_{\mathrm{acc}} L^{\dagger}-T_{\mathrm{visc}}\right)=4 \pi r\left[\left(\dot{m}_{\mathrm{jet}}+F_{\mathrm{rad}}\right) L^{\dagger}+S_{\mathrm{L}}\right],
\end{aligned}
$$

where $T_{\text {visc }}$ and $F_{\text {rad }}$ are the internal viscous torque and the energy flux radiated away from the surface of disk, respectively.

In Eqs. (16) and (17), $E^{\dagger}$ and $L^{\dagger}$ are the specific energy and angular momentum of the disk matter, being expressed by (Novikov \& Thorne 1973)

$$
\begin{aligned}
E^{\dagger} & =\left(1-2 \chi^{-2}+a_{*} \chi^{-3}\right) /\left(1-3 \chi^{-2}+2 a_{*} \chi^{-3}\right)^{1 / 2}, \\
L^{\dagger} & =M \chi\left(1-2 a_{*} \chi^{-3}+a_{*}^{2} \chi^{-4}\right) /\left(1-3 \chi^{-2}+2 a_{*} \chi^{-3}\right)^{1 / 2},
\end{aligned}
$$

where $\chi \equiv \sqrt{r / M}=\xi^{1 / 2} \chi_{\mathrm{ms}}$, and the quantities $L^{\dagger}$ and $E^{\dagger}$ are related by

$\frac{\mathrm{d} E^{\dagger}}{\mathrm{d} r}=\Omega_{\mathrm{D}} \frac{\mathrm{d} L^{\dagger}}{\mathrm{d} r}$.

The terms on the left hand side of Eqs. (16) and (17) are the radial transfer of energy and angular momentum due to disk accretion, respectively, while the terms on the right-hand side of these equations represent the transfer of energy and angular momentum due to disk radiation and jet.

Not long ago, some authors (Balbus \& Hawley 1998; Agol \& Krolik 2000) pointed out that the magnetic stresses might exert a time-steady torque on the inner edge of the disk, and a nonzero torque at $r_{\mathrm{ms}}$ can be expressed as follows,

$T_{\mathrm{ms}} \approx \frac{\left(B_{\mathrm{ms}}^{\mathrm{p}}\right)^{2}}{4 \pi} 4 \pi H_{\mathrm{ms}} r_{\mathrm{ms}}^{2}=0.2 \dot{M}_{\mathrm{acc}}\left(r_{\mathrm{ms}}\right) \sqrt{r_{\mathrm{ms}} r_{\mathrm{H}}}$,

where $B_{\mathrm{ms}}^{\mathrm{p}}, T_{\mathrm{ms}}$, and $H_{\mathrm{ms}}$ are the poloidal magnetic field, torque, and the height of the disk at the inner edge of disk, respectively, and $\left(H / r_{\mathrm{ms}}\right)_{\max } \approx 0.1$ is assumed in calculations. Equations (1) and (4) are used in the last step of deriving Eq. (21).

Incorporating Eqs. (16)-(20), we have

$$
\begin{aligned}
F_{\mathrm{rad}}= & \left.-\frac{\mathrm{d} \Omega_{\mathrm{D}} / \mathrm{d} r}{4 \pi r}\left(E^{\dagger}-\Omega_{\mathrm{D}} L^{\dagger}\right)\right)^{-2} \\
& \times\left[\left(\int_{r_{\mathrm{ms}}}^{r}\left(E^{\dagger}-\Omega_{\mathrm{D}} L^{\dagger}\right)\left(\dot{M}_{\mathrm{acc}} \frac{\mathrm{d} L^{+}}{\mathrm{d} r}\right)+\left(E_{\mathrm{ms}}^{\dagger}-\Omega_{\mathrm{ms}} L_{\mathrm{ms}}^{\dagger}\right) T_{\mathrm{ms}}\right)\right. \\
& \left.-\int_{r_{\mathrm{ms}}}^{r}\left(E^{\dagger}-\Omega_{\mathrm{D}} L^{\dagger}\right) 4 \pi r S_{\mathrm{L}} \mathrm{d} r\right] .
\end{aligned}
$$

The quantity $F_{\text {rad }}$ in Eq. (22) is the disk radiation flux in the presence of the jet, while the first integral on the right hand side of the equation represents the release rate of the accreting matter's
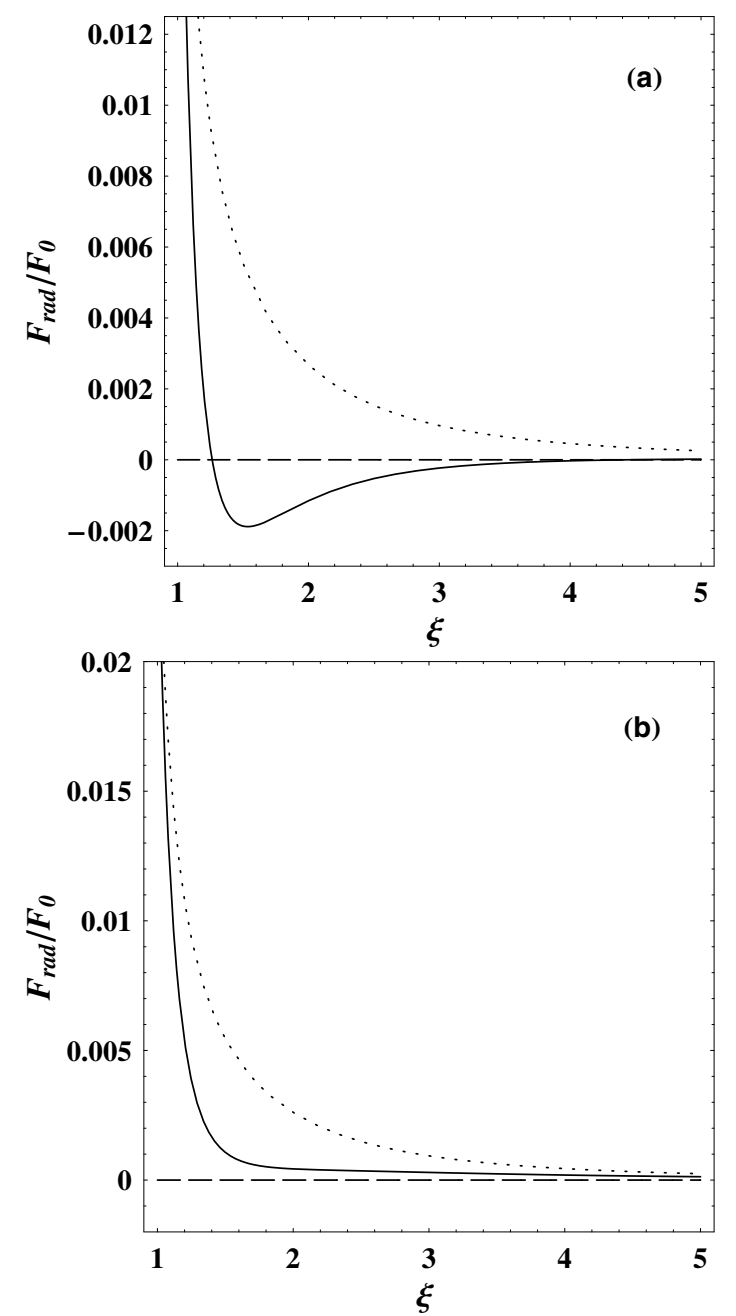

Fig. 4. The curves of $F_{\text {rad }} / F_{0}$ versus $\xi$ for a) $a_{*}=0$ and $\alpha=4$; b) $a_{*}=0.95$ and $\alpha=5$. The solid and dotted lines represent the disk radiation flux with and without jet, respectively. The parameter $F_{0}=$ $\dot{M}_{\mathrm{acc}}\left(r_{\mathrm{ms}}\right) /\left(r_{\mathrm{ms}}^{2} M^{2}\right)$ is defined, and $\gamma_{\mathrm{j}}=10$ are assumed.

energy, in which a magnetic torque exerted at ISCO is taken into account, and the second integral is the cooling rate due to the outflow/jet driven by the BP process, in which the kinetic flux and Poynting flux are included. It is expected that some relation between the BP power and disk luminosity can be obtained based on our model.

\section{Relationship between jet power and disk luminosity}

The relationship between the BP power and disk luminosity can be discussed based on Eq. (22), and the curves of $F_{\text {rad }} / F_{0}$ versus $\xi$ for the given values of $a_{*}, \gamma_{\mathrm{j}}$, and $\alpha$ are shown in Fig. 4, in which the disk radiation flux is significantly reduced due to the existence of the jet driven by the BP process. This result is consistent with ones from other authors (Donea \& Biermann 1996; Knigge 1999; Kuncic \& Bicknell 2007).

From Fig. 4a we find that the radiation flux at the presence of the jet could become negative in the inner disk, and this result is unphysical. Inspecting Eq. (21), we find that this unphysical result can be removed, provided that the cooling contribution of 


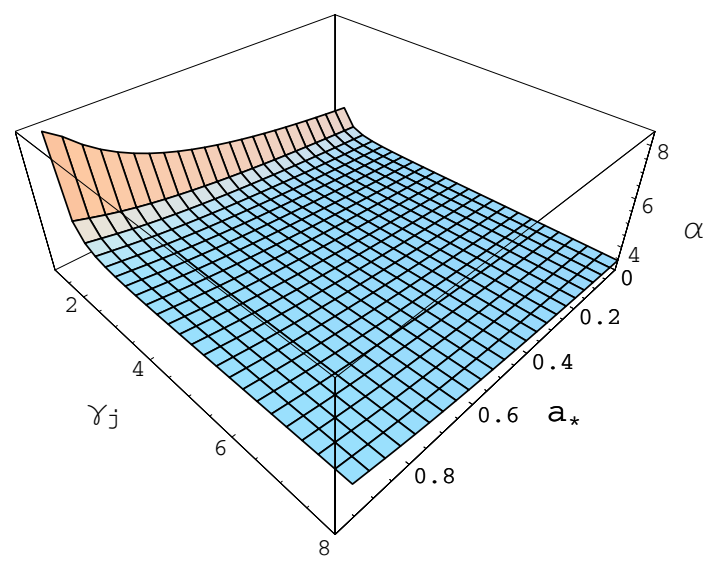

Fig. 5. The contour surface of $\left(F_{\text {rad }}\right)_{\min }=0$ in the 3-dimensional parameter space $\left(a_{*}, \alpha, \gamma_{\mathrm{j}}\right)$.

the BP process is not very strong. The following condition is required by non-negative radiation flux:

$\left(F_{\text {rad }}\right)_{\min } \geq 0$,

where $\left(F_{\mathrm{rad}}\right)_{\min }$ is the minimum disk radiation at the presence of the jet.

Combining Eq. (23) with (22), we have the contour surface of $\left(F_{\mathrm{rad}}\right)_{\min }=0$ in the 3 -dimensional parameter space $\left(a_{*}, \alpha, \gamma_{\mathrm{j}}\right)$ as shown in Fig. 5, in which the values of the parameters above the contour surface $\left(F_{\mathrm{rad}}\right)_{\min }=0$ correspond to positive radiation flux, while those below the surface are unphysical.

Inspecting Fig. 5, we find that the requirement for nonnegative radiation flux is very sensitive to the self-similar index $\alpha$, which should be greater than some critical value for the given black hole spin $a_{*}$ and Lorentz factor $\gamma_{\mathrm{j}}$ of the jet. This result implies that the poloidal magnetic field expressed by Eq. (2) should reduce steeply enough with the increasing cylindrical radius to avoid an unphysical disk flux.

Taking the nonzero torque exerted at $r_{\mathrm{ms}}$ into account, we have the disk luminosity by integrating Eq. (16) as

$$
\begin{aligned}
L_{\mathrm{disk}} & =\int_{r_{\mathrm{ms}}}^{r_{\text {out }}} 4 \pi r F_{\mathrm{rad}} E^{\dagger} \mathrm{d} r=\int_{r_{\mathrm{ms}}}^{r_{\text {out }}} \dot{M}_{\mathrm{acc}}(r) \mathrm{d} E^{\dagger} \\
& -4 \pi \int_{r_{\mathrm{ms}}}^{r_{\text {out }}} S_{\mathrm{L}} \Omega_{\mathrm{D}} r \mathrm{~d} r+T_{\mathrm{ms}} \Omega_{\mathrm{D}} .
\end{aligned}
$$

And the BP power can be expressed as

$P_{\mathrm{BP}}=\int_{r_{\mathrm{ms}}}^{r_{\text {out }}} 4 \pi r\left(\dot{m}_{\mathrm{jet}} E^{\dagger}+S_{\mathrm{L}} \Omega_{\mathrm{D}}\right) \mathrm{d} r$

where the kinetic and electromagnetic energy are included. Inspecting Eqs. (24) and (25), we find that the ratio of the BP power to the disk luminosity depends on the parameters $a_{*}, \alpha$, and $\gamma_{\mathrm{j}}$, and the curves of $\log \left(P_{\mathrm{BP}} / L_{\text {disk }}\right)$ versus $\gamma_{\mathrm{j}}$ with different values of $a_{*}$ and $\alpha$ are shown in Fig. 6.

As shown in Fig. 6, the ratio $P_{\mathrm{BP}} / L_{\text {disk }}$ decreases with the Lorentz factors $\gamma_{j}$ very steeply for $\gamma_{j}<3$, while it almost remains constant for $\gamma_{\mathrm{j}} \geq 3$. And the ratio decreases with the increasing self-similar index $\alpha$, while it increases with the black hole spin $a_{*}$. These results are consistent with those given in C02, except that the ratios obtained in our model are greater than those given in $\mathrm{C} 02$ for the same values of $a_{*}, \alpha$, and $\gamma_{\mathrm{j}}$. The difference between the two models might arise from the influence of the BP process on the disk radiation and the contribution of Poynting flux in the jet being not taken into account in $\mathrm{C} 02$.
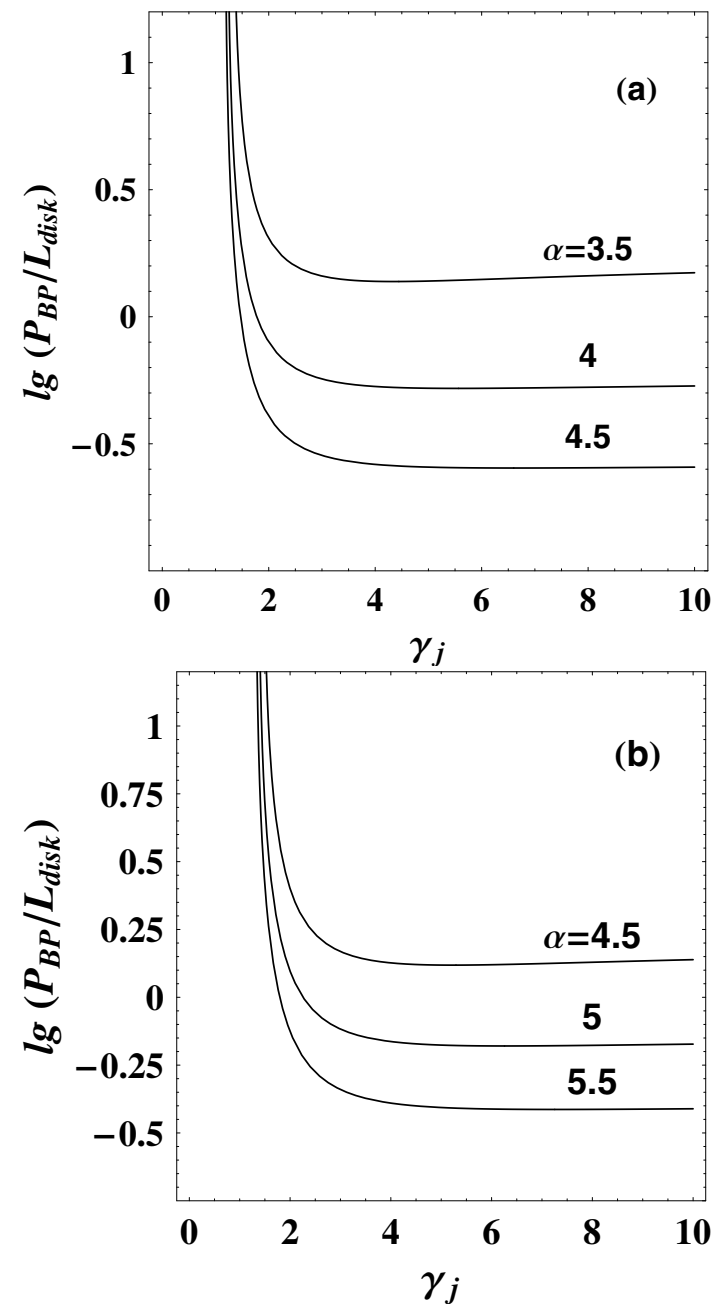

Fig. 6. The curves of $\log \left(P_{\mathrm{BP}} / L_{\mathrm{disk}}\right)$ versus $\gamma_{\mathrm{j}}$ for different values of $\alpha$ with a) $a_{*}=0$ and b) $a_{*}=0.95$.

McKinney (2006) proposes that AGNs are observed to have jets with Lorentz factor $\sim 10$ (Urry \& Padovani 1995; Biretta et al. 1999). Considering that $P_{\mathrm{BP}} / L_{\mathrm{disk}}$ is insensitive to the Lorentz factor for $\gamma_{j} \geq 3$, we take $\gamma_{j}=10$ when studying the variation in $P_{\mathrm{BP}} / L_{\text {disk }}$ with the two parameters $a_{*}$ and $\alpha$. As shown in Fig. 7, the contours of $P_{\mathrm{BP}} / L_{\text {disk }}=0.1,1$, and 10 , and the contour of $\left(F_{\mathrm{rad}}\right)_{\min }=0$ are plotted in $a_{*}-\alpha$ parameter space, by which the parameter space are divided into regions I, II, III, and IV.

In region I the disk luminosity dominates the BP power significantly, while the BP power is comparable to the disk luminosity in regions II and III, in which the radiation cooling mode coexists with outflow/jet cooling mode. The BP power is less and greater than the disk luminosity, in regions II and III, respectively. Region IV is indicated as a forbidden region, which corresponds to the negative radiation flux. The contour of $P_{\mathrm{BP}} / L_{\mathrm{disk}}=10$ lies in the region IV, which means that the positive radiation flux requires the BP power not to be much greater than the disk luminosity. Comparing with the results given in $\mathrm{C} 02$, the larger self-similar index $\alpha$ is required by the positive disk radiation flux.

To fit the relativistic jet power from AGNs based on the magnetic field configuration depicted in Fig. 1, we should compare the importance of the $\mathrm{BZ}$ power with respect to the $\mathrm{BP}$ power. Incorporating Eqs. (5) and (25), we have the contours of $P_{\mathrm{BZ}} / P_{\mathrm{BP}}=$ const. in the $a_{*}-\alpha$ parameter space with $\gamma_{\mathrm{j}}=10$ 


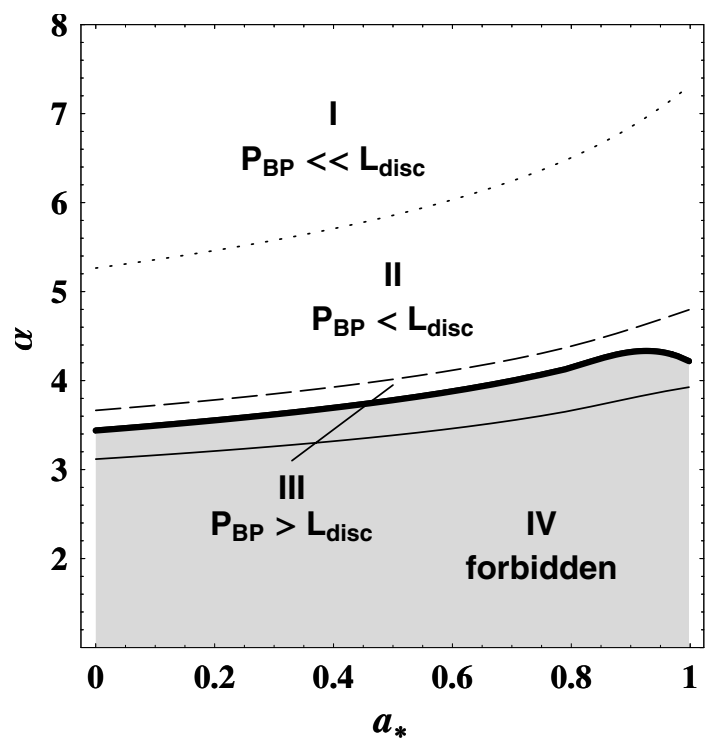

Fig. 7. The $a_{*}-\alpha$ parameter space: the contours of $P_{\mathrm{BP}} / L_{\mathrm{disk}}=0.1$, 1 , and 10 correspond respectively to the dotted, dashed and thin-solid lines, respectively, and the contour of $\left(F_{\mathrm{rad}}\right)_{\min }=0$ is a thick solid line for $\gamma_{\mathrm{j}}=10$.

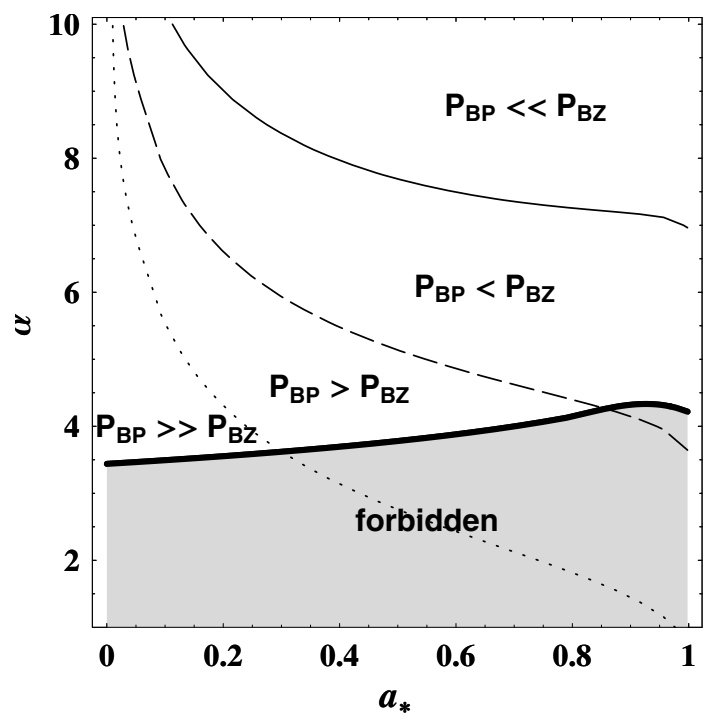

Fig. 8. The $a_{*}-\alpha$ parameter space: the contours of $P_{\mathrm{BZ}} / P_{\mathrm{BP}}=0.1$, 1 , and 10 correspond respectively to the dotted, dashed, and thin-solid lines, and the contour of $\left(F_{\text {rad }}\right)_{\min }=0$ is a thick solid line for $\gamma_{\mathrm{j}}=10$.

as shown in Fig. 8, in which $P_{\mathrm{BZ}} / P_{\mathrm{BP}}=0.1,1$, and 10 , respectively. The shaded region below the thick solid line represents the forbidden region as argued above. The region between the dotted and thin solid lines indicates $0.1<P_{\mathrm{BZ}} / P_{\mathrm{BP}}<10$, i.e., the $\mathrm{BZ}$ power is comparable to the $\mathrm{BP}$ power. The region above the thin solid line and the one below the dotted line represent $P_{\mathrm{BP}} \ll P_{\mathrm{BZ}}$ and $P_{\mathrm{BP}} \gg P_{\mathrm{BZ}}$, respectively. Thus the contribution of the BZ and BP processes to the jet power from AGNs can be determined by the values of the parameters $\alpha$ and $a_{*}$.

Based on the above discussion, we find that both the BZ and BP powers should be taken into account in fitting the relativistic jet power, provided that the values of $a_{*}$ and $\alpha$ are taken in the region between the dotted and thin-solid lines as shown in Fig. 8.

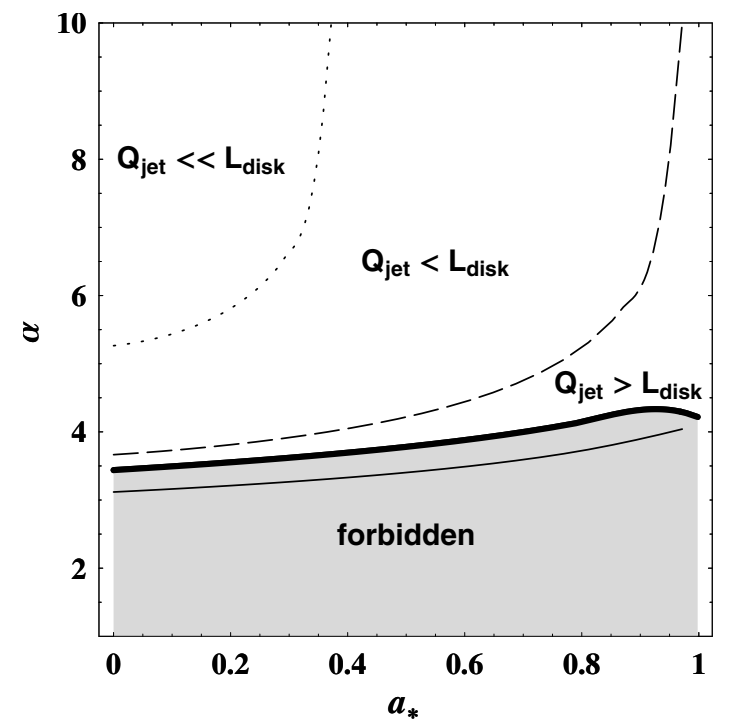

Fig. 9. The $a_{*}-\alpha$ parameter space- The contours of $Q_{\text {jet }} / L_{\text {disk }}=0.1$, 1 and 10 correspond respectively to the dotted, dashed, and thin-solid lines, and the contour of $\left(F_{\mathrm{rad}}\right)_{\min }=0$ is a thick solid line for $\gamma_{\mathrm{j}}=10$.

Thus the jet power can be fitted as the sum of the BZ and BP powers,

$Q_{\text {jet }}=P_{\mathrm{BP}}+P_{\mathrm{BZ}}$

To study the relationship between the jet power and the disk luminosity, we plot the contours of $Q_{\text {jet }} / L_{\text {disk }}=$ constant in $a_{*}-\alpha$ parameter space with $\gamma_{\mathrm{j}}=10$ as shown in Fig. 9.

It is found from Fig. 9 that the jet power and the disk luminosity are comparable, except that the black hole rotates slowly and the magnetic field decreases very steeply with the cylindrical radius. The region above the thin solid line in the $a_{*}-\alpha$ parameter space represents $Q_{\text {jet }} \ll L_{\text {disk }}$. As shown in Fig. 9, the contour of $Q_{\text {jet }} / L_{\text {disk }}=10$ lies in the forbidden region, and it implies that the jet power cannot be significantly greater than the disk luminosity, which is required by non-negative disk flux.

According to MT03 the disk luminosity is related to the broad-line region luminosity by $L_{\mathrm{BLR}} \approx 0.1 L_{\mathrm{disk}}$. Taking the accretion rate at ISCO as $\dot{M}_{\text {acc }}\left(r_{\mathrm{ms}}\right)=0.1 \dot{M}_{\text {Edd }}$, we derive the jet power and broad-line region luminosity of 11 FSRQs and 17 SSRQs based on Eqs. (5), (24), (25), and (26) as shown in Table 1 . In addition, we mark the points of values of $a_{*}$ and $\alpha$ corresponding to these sources in the $a_{*}-\alpha$ parameter space as shown in Fig. 10.

It is found from Fig. 10 that the parameters $a_{*}$ and $\alpha$ of 25 samples (except one SSRQ and two FSRQs) fall in the region between the dotted and thick-solid lines, which indicates that the jet power is comparable to the disk luminosity. This result is consistent with what was derived by MT03 and D'Elia et al. (2003). Other sources given in L06 cannot be fitted by our model for the following reasons: (1) the constraint of non-negative disk radiation flux expressed by Eq. (23), and (2) the accretion rate at ISCO is assumed to be 0.1 Eddington accretion rate. Some sources with disk luminosity exceeding this limit are not fitted clearly.

\section{Conclusions}

The coupling of disk-jet is essential for jet production from AGNs and stellar black hole systems (Blandford 2002). The BZ 
Table 1. The values of the concerned parameters for fitting the jet power and broad-line region luminosity and black hole mass of 11 FSRQs and 17 SSRQs, where $\gamma_{\mathrm{j}}=10$ and $\dot{M}_{\mathrm{acc}}\left(r_{\mathrm{ms}}\right)=0.1 \dot{M}_{\text {Edd }}$ are assumed.

\begin{tabular}{|c|c|c|c|c|c|c|c|}
\hline Sources & $\log Q_{\text {jet }}$ & $\log L_{\mathrm{BLR}}$ & $\log M_{\mathrm{BH}}$ & $a_{*}$ & $\alpha$ & $\log P_{\mathrm{BZ}}$ & $\log P_{\mathrm{BP}}$ \\
\hline $0017+154^{\mathrm{S}}$ & $47.19^{\text {[BM87] }}$ & $45.79^{[\mathrm{CIV}, \mathrm{C} 91]}$ & $9.62^{[\mathrm{MgII}, \mathrm{H} 02]}$ & 0.89 & 4.51 & 46.94 & 46.83 \\
\hline $0022+297^{\mathrm{S}}$ & $45.61^{[\mathrm{K} 98]}$ & $44.15^{[\mathrm{H} \beta, \mathrm{S} 93]}$ & $7.81^{[\mathrm{H} \beta, \mathrm{G} 01]}$ & 0.98 & 4.81 & 45.44 & 45.13 \\
\hline $0119-046^{\mathrm{S}}$ & $46.52^{[\mathrm{R} 99]}$ & $46.00^{[\mathrm{MgII}, \mathrm{SS} 91]}$ & $9.91^{[\mathrm{MgII}, \mathrm{B} 94]}$ & 0.25 & 4.64 & 45.74 & 46.44 \\
\hline $0134+329^{S}$ & $46.30^{[\mathrm{BM} 87]}$ & $44.96^{[\mathrm{H} \beta, \mathrm{JB} 91]}$ & $8.65^{[\mathrm{H} \beta, \mathrm{C} 97]}$ & 0.94 & 4.90 & 46.12 & 45.83 \\
\hline $0238+100^{\mathrm{S}}$ & $46.10^{[\mathrm{N} 89]}$ & 45.59 [CIV,C91] & $9.48^{[\mathrm{CIV}, \mathrm{C} 91]}$ & 0.29 & 4.76 & 45.46 & 45.99 \\
\hline $0336-019^{\mathrm{F}}$ & $45.18^{[\mathrm{BM} 87]}$ & $45.00^{[\mathrm{H} / \mathrm{JB} 91]}$ & $8.89^{[\mathrm{H} \beta, \mathrm{G} 01]}$ & 0.13 & 5.12 & 44.18 & 45.13 \\
\hline $0403-132^{\mathrm{F}}$ & $45.60^{[\mathrm{BM} 87]}$ & $45.25^{[\mathrm{H} \beta, \mathrm{O} 84]}$ & $9.08^{[\mathrm{H} \beta, \mathrm{M} 96]}$ & 0.35 & 5.40 & 45.23 & 45.36 \\
\hline $0607-157^{\mathrm{F}}$ & $44.20^{[\mathrm{BM} 87]}$ & $43.56^{[\mathrm{H} \beta, \mathrm{H} 78]}$ & $7.32^{[\mathrm{H} \beta, \mathrm{G} 01]}$ & 0.58 & 5.55 & 44.01 & 43.75 \\
\hline $0637-752^{\mathrm{F}}$ & $46.48^{[\mathrm{CJ} 01]}$ & $45.44^{[\mathrm{H} \beta, \mathrm{T} 93]}$ & $8.81^{[\mathrm{H} \beta, \mathrm{G} 01]}$ & 0.98 & 9.15 & 46.47 & 44.85 \\
\hline $0837-120^{\mathrm{S}}$ & $44.79^{[\mathrm{R} 99]}$ & $45.00^{[\mathrm{H} \beta, \mathrm{B} 96]}$ & $8.86^{[\mathrm{H} \beta, \mathrm{B} 96]}$ & 0.12 & 6.01 & 44.06 & 44.70 \\
\hline $0838+133^{\mathrm{F}}$ & $46.19^{\text {[BM87] }}$ & $45.14^{[\mathrm{H} \beta, \mathrm{JB} 91]}$ & $8.67^{[H \beta, B 96]}$ & 0.94 & 6.38 & 46.12 & 45.36 \\
\hline $0903+169^{S}$ & $45.30^{[\mathrm{BM} 87]}$ & $44.69^{[\mathrm{H} \beta, \mathrm{B} 96]}$ & $8.39^{[\mathrm{H} \beta, \mathrm{B} 96]}$ & 0.65 & 6.46 & 45.20 & 44.56 \\
\hline $1023+067^{S}$ & $46.50^{[\mathrm{BM} 87]}$ & $45.07^{[\mathrm{MgII}, \mathrm{C} 91]}$ & $8.99^{[\mathrm{MgII}, \mathrm{C} 91]}$ & 0.85 & 4.29 & 46.20 & 46.20 \\
\hline $1040+123^{S}$ & $46.27^{\text {[BM87] }}$ & $45.11^{[\mathrm{MgII}, \mathrm{N} 79]}$ & $8.76^{[\mathrm{MgII}, \mathrm{H} 02]}$ & 0.91 & 5.29 & 46.12 & 45.73 \\
\hline $1250+568^{\mathrm{S}}$ & $45.50^{[\mathrm{BM} 87]}$ & $44.57^{[\mathrm{H} \beta, \mathrm{JB} 91]}$ & $8.31^{[\mathrm{H} \beta, \mathrm{B} 96]}$ & 0.74 & 5.18 & 45.30 & 45.06 \\
\hline $1253-055^{\mathrm{F}}$ & $45.70^{[\mathrm{BM} 87]}$ & $44.64^{[\mathrm{H} \beta, \mathrm{M} 96]}$ & $8.28^{[\mathrm{H} \beta, \mathrm{G} 01]}$ & 0.88 & 5.54 & 45.11 & 45.57 \\
\hline $1318+113^{\mathrm{S}}$ & $46.86^{[\mathrm{G} 91]}$ & $45.86^{[\mathrm{CIV}, \mathrm{C} 91]}$ & $9.32^{[\mathrm{CIV}, \mathrm{C} 91]}$ & 0.95 & 7.80 & 46.83 & 45.63 \\
\hline $1334-127^{\mathrm{F}}$ & $44.91[\mathrm{CJ} 01]$ & $44.18^{[\mathrm{MgII}, \mathrm{S} 93]}$ & $7.98^{[\mathrm{MgII}, W 86]}$ & 0.57 & 5.08 & 44.64 & 44.58 \\
\hline $1442+101^{\mathrm{S}}$ & $46.95^{[B M 87]}$ & $45.93^{[\mathrm{CIV}, \mathrm{C} 91]}$ & $9.93^{[\mathrm{H} \beta, \mathrm{H} 03]}$ & 0.44 & 4.08 & 46.31 & 46.84 \\
\hline $1559+173^{S}$ & $46.81^{[S 90]}$ & $45.66^{[\mathrm{MgII}, \mathrm{C} 91]}$ & $9.25^{[\mathrm{MgII}, \mathrm{C} 91]}$ & 0.93 & 5.59 & 46.69 & 46.18 \\
\hline $1606+289^{S}$ & $46.56^{[\mathrm{BM} 87]}$ & $45.61[\mathrm{CIV}, \mathrm{C} 91]$ & $9.37[\mathrm{CIV}, \mathrm{C} 91]$ & 0.73 & 5.04 & 46.34 & 46.16 \\
\hline $1622+238^{S}$ & $46.48^{[\mathrm{BM} 87]}$ & $45.34^{[\mathrm{MgII}, \mathrm{SS} 91]}$ & $9.53^{[\mathrm{H} \beta, \mathrm{B} 96]}$ & 0.16 & 3.63 & 44.97 & 46.47 \\
\hline $1641+399^{\mathrm{F}}$ & $45.30^{[\mathrm{BM} 87]}$ & $45.47^{[\mathrm{H} \beta, \mathrm{L} 96]}$ & $9.27^{[\mathrm{H} \beta, \mathrm{M} 96]}$ & 0.29 & 8.07 & 45.24 & 44.39 \\
\hline $1954-388^{\mathrm{F}}$ & $44.12^{[\mathrm{CJ} 01]}$ & $44.20^{[\mathrm{H} \beta, \mathrm{T} 93]}$ & $7.99^{[\mathrm{H} \beta, \mathrm{O} 02]}$ & 0.32 & 8.11 & 44.07 & 43.14 \\
\hline $1954+513^{\mathrm{F}}$ & $46.04^{[\mathrm{K} 90]}$ & $45.39^{[\mathrm{Mg} ?, \mathrm{~L} 96]}$ & $9.18^{[\mathrm{MgII}, \mathrm{L} 96]}$ & 0.54 & 5.27 & 45.79 & 45.68 \\
\hline $1655+077^{F}$ & $45.00^{[\mathrm{M} 93]}$ & $43.62^{[\mathrm{MgII}, \mathrm{W} 86]}$ & $7.28^{[\mathrm{MgII}, \mathrm{W} 86]}$ & 0.96 & 4.92 & 44.83 & 44.51 \\
\hline $2120+168^{S}$ & $46.88^{[\text {[BM87] }}$ & $45.57^{[\mathrm{CIV}, \mathrm{O} 94]}$ & $9.68^{[\mathrm{MgII}, \mathrm{H} 02]}$ & 0.54 & 3.85 & 46.28 & 46.76 \\
\hline $2354+144^{\mathrm{S}}$ & $46.73^{[\mathrm{H} 83]}$ & $44.75^{[\mathrm{H} \beta, \mathrm{C} 91]}$ & $9.37^{[\mathrm{H} \beta, \mathrm{C} 91]}$ & 0.87 & 5.91 & 46.63 & 46.06 \\
\hline
\end{tabular}

Notes: Column (1): IAU source name. The superscript "F" and "S" represent FSRQs and SSRQs, respectively. Column (2): jet power Q units of erg s ${ }^{-1}$. The superscript represents references of radio extended flux density in calculating the jet power. Column (3): broad-line region luminosity in units of $\mathrm{erg} \mathrm{s}^{-1}$. The superscript represents the adopted lines in calculating broad-line region luminosity and references for lines. Column (4): black hole mass in units of $M_{\odot}$. The superscript represents lines for estimating black hole mass and references for lines. Column (5): the fitted black hole spin. Column (6): the fitted self-similar index. Column (7): the corresponding BZ power in units of erg s${ }^{-1}$. Column (8): the corresponding BP power in units of e erg s ${ }^{1}$.

References: B94: Brotherton et al. (1994). B96: Brotherton (1996). BM87: Browne \& Murphy (1987). C91: Corbin (1991). C97: Corbin (1997). CJ01: Cao \& Jiang (2001). G01: Gu et al. (2001). H78: Hunstead et al. (1978). H83: Hintzen et al. (1983). H02: Hough et al. (2002). H03: Hirst et al. (2003). JB91: Jackson \& Browne (1991). K90: Kollgaard et al. (1990). K98: Kapahi et al. (1998). L96: Lawrence et al. (1996). M93: Murphy et al. (1993). M96: Marziani et al. (1996). N79: Neugebauer et al. (1979). N89: Neff et al. (1989). O84: Oke et al. (1984). O94: Osmer et al. (1994). O02: Oshlack et al. (2002). R99: Reid et al. (1999). S90: Saikia et al. (1990). S93: Stickel et al. (1993). SS91: Steidel \& Sargent (1991). T93: Tadhunter et al. (1993). W86: Wilkes (1986).

and BP processes are two main mechanisms for driving the jets. In this paper a simplified model of jet power from AGNs is proposed by combining the $\mathrm{BZ}$ and $\mathrm{BP}$ processes with the disk accretion. The expressions of the BP power and disk luminosity can be derived from the conservation laws of mass, angular momentum, and energy. These equations consist of a closed set for resolving the BP power and the disk luminosity. It turns out that the relative importance of a series of quantities related to the jet power, such as the BP power, the BZ power, the disk luminosity, and the jet power itself can be displayed visually in the $a_{*}-\alpha$ parameter space.

It is shown that the disk radiation flux and luminosity decrease due to a fraction of the accretion energy being channelled into the outflow/jet in the BP process, and the dominant cooling mode of the accretion disk is determined mainly by how the the poloidal magnetic field decreases with the cylindrical radius of the jet. The dominant mode is radiation cooling for the magnetic field decreasing very steeply with the cylindrical radius, while the mode could be outflow/jet cooling for the magnetic fields decreasing less steeply. However, the strength of the outflow/jet cooling should be less than some critical values to avoid a negative disk radiation.

In this model the jet power is regarded as the sum of the BZ and BP powers, which are related to the disk accretion rate by assuming a relation connecting the magnetic field at the black hole horizon with the accretion rate at ISCO [given by Eq. (4)], and the broad-line region luminosity is assumed to be one tenth of the disk luminosity. Based on these assumptions, we fit the jet power and broad-line region luminosity of 11 FSRQs and 17 SSRQs, whose jet power is almost the same as the disk luminosity.

In this simplified model, the poloidal magnetic field is assumed to be anchored in a thin disk, scaling with the disk radius in a power law, and the variation of the poloidal magnetic with the cylindrical radius is described by a self-similar index given by $\mathrm{C} 02$. As a matter of fact, the magnetic field configurations could be much more complicated, and the accretion mode can affect the jet power and disk luminosity significantly. We shall 


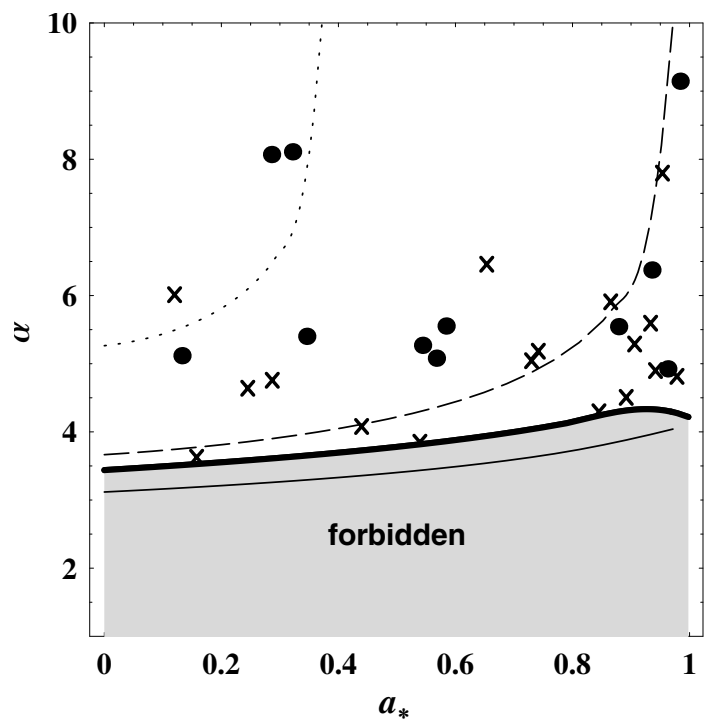

Fig. 10. The $a_{*}-\alpha$ parameter space- The values of the parameters $a_{*}$ and $\alpha$ correspond to the jet power and the disk luminosity of 11 FSRQs (solid circles), 17 SSRQs (crosses) listed in Table 1, and the contours of $Q_{\text {jet }} / L_{\text {disk }}=0.1,1$, and 10 correspond respectively to the dotted, dotdashed, dashed, and thin-solid lines, and the contour of $\left(F_{\text {rad }}\right)_{\min }=0$ is a thick solid line for $\gamma_{\mathrm{j}}=10$.

improve our model by combining different magnetic field configurations with inefficient accretion mode, such as ADAF, to fit the observations of the AGNs with strongly dominated jet power in our future work.

Acknowledgements. This work is supported by the National Natural Science Foundation of China under grants 10573006 and 10778712.

\section{References}

Agol, E., \& Krolik J. H. 2000, ApJ, 528, 161

Amitage, P. J. 1998, ApJ, 501, 189

Balbus, S. A., \& Hawley, J. F. 1998, Rev. Mod. Phys., 70, 1

Biretta, J. A., Sparks, W. B., \& Macchetto, F. 1999, ApJ, 520, 621

Blandford, R. D. 2002, Lighthouses of the Universe: The Most Luminous Celestial Objects and Their Use for Cosmology Proceedings of the MPA/ESO/, 381 [arXiv: astro-ph/0202265]

Blandford, R. D., \& Znajek, R. L. 1977, MNRAS, 179, 433

Blandford, R. D., \& Payne, D. G. 1982, MNRAS, 199, 883

Brotherton, M. S. 1996, ApJS, 102, 1 (B96)

Brotherton, M. S., Wills, B. J., Steidel, C. C., et al. 1994, ApJ, 423, 131 (B94)

Browne, I. W. A., \& Murphy, D. W. 1987, MNRAS, 226, 601 (BM87)

Camenzid, M. 1986, A\&A, 156, 137

Cao, X. W. 2002, MNRAS, 332, 999 (C02)

Cao, X. W., \& Jiang, D. R. 2001, MNRAS, 320, 347 (CJ01)

Celotti, A., Padovani, P., \& Ghisellini, G. 1997, MNRAS, 286, 415

Corbin, M. R. 1991, ApJ, 375, 503 (C91)

Corbin, M. R. 1997, ApJS, 113, 245 (C97)
D'Elia, V., Padovani, P., \& Landt, H. 2003, MNRAS, 339, 1081

Donea, A. C., \& Biermann, P. L. 1996, A\&A, 316, 43

Ghosh, P., \& Abramowicz, M. A. 1997, MNRAS, 311, 576

Gu, M. F., Cao, X. W., \& Jiang, D. R. 2001, MNRAS, 327, 1111 (G01)

Hawley, J. F., Gammie, C. F., \& Balbus, S. A. 1995, ApJ, 440, 742

Hintzen, P., Ulvestad, J., \& Owen, F. 1983, AJ, 88, 709 (H83)

Hirst, P., Jackson, N., \& Rawlings, S. 2003, MNRAS, 346, 1009 (H03)

Hough, D. H., Vermeulen, R. C., Readhead, A. C. S., et al. 2002, AJ, 123, 1258 ( $\mathrm{H} 02$ )

Hunstead, R. W., Murdoch, H. S., \& Shobbrook, R. R. 1978, MNRAS, 185, 149 (H78)

Jackson, N., \& Browne, I. W. A. 1991, MNRAS, 250, 414 (JB91)

Kollgaard, R. I., Wardle, J. F. C., \& Roberts, D. H. 1990, AJ, 100, 1057 (K90)

Kapahi, V. K., Athreya, R. M., Subrahmanya, C. R., et al. 1998, ApJS, 118, 327 (K98)

Knigge, C. 1999, MNRAS, 309, 409

Kuncic, Z., \& Bicknell, C. V. 2007, Mod. Phys. Lett. A, accepted [arXiv: 0707.1914v1]

Lawrence, C. R., Zucker, J. R., Readhead, A. C. S., et al. 1996, ApJS, 107, 541 (L96)

Lovelace, R. V. E., Romanova, M. M., \& Newman, W. I. 1994, ApJ, 437, 136

Liu, Y., Jiang, D. R., \& Gu, M. F. 2006, ApJ, 637, 669 (L06)

Livio, M. 1999, Phys. Rep., 311, 225

Livio, M., Ogilvie, G. I., \& Pringle, J. E. 1999, ApJ, 512, 100

Lubow, S. H., Papaloizou, J. C. B., \& Prigle, J. E. 1994, MNRAS, 268, 1010

Macdonald, D., \& Thorne, K. S. 1982, MNRAS, 198, 345

Maraschi, L., \& Tavecchio, F. 2003, ApJ, 593, 667 (MT03)

Marziani, P., Sulentic, J. W., Dultzin-Hacyan, et al. 1996, ApJS, 104, 37 (M96)

McKinney, J. C. 2006, MNRAS, 368, 1561

McLure, R. J., \& Dunlop, J. S. 2001, MNRAS, 327, 199

McLure, R. J., \& Jarvis, M. J. 2002, MNRAS, 337, 109

Miller, J. M., Raymond, J., Fabian, A., et al. 2006, Nature, 441, 953

Moderski, R., Sikora, M., \& Lasota, J. P. 1997, in Relativistic Jets in AGNs, ed. M. Ostrowski, M. Sikora, G. Madejski, \& M. Belgelman, Krakow, 110 [arXiv: 0707.1914v]

Murphy, D. W., Browne, I. W. A., \& Perley, R. A. 1993, MNRAS, 264, 298 (M93)

Neff, S. G., Hutchings, J. B., \& Gower, A. C. 1989, AJ, 97, 1291 (N89)

Novikov, I. D., Thorne, K. S. 1973, in Black Holes, ed. C. Dewitt, (New York: Gordon and Breach), 345

Neugebauer, G., Oke, J. B., Becklin, E. E., \& Matthews, K. 1979, ApJ, 230, 79 (N79)

Oke, J. B., Shields, G. A., \& Korycansky, D. G. 1984, ApJ, 277, 64 (O84)

Oshlack, A. Y. K. N., Webster, R. L., \& Whiting, M. T. 2002, ApJ, 576, 81 (O02)

Osmer, P. S., Porter, A. C., \& Green, R. F. 1994, ApJ, 436, 678 (O94)

Punsly, B. 2005, ApJ, 623, L9

Reid, R. I., Kronberg, P. P., \& Perley, R. A. 1999, ApJS, 124, 285 (R99)

Saikia, D. J., Junor, W., Cornwell, T. J., et al. 1990, MNRAS, 245, 408 (S90)

Steidel, C. C., \& Sargent, W. L. W. 1991, ApJ, 382, 433 (SS91)

Stickel, M., Kühr H., \& Fried, J. W. 1993, A\&AS, 97, 483 (S93)

Spruit, H. C. 1996, Evolutionary Processes in Binary Stars, NATO ASIC Proc. 477, 249, see [arXiv: astro-ph/9602022]

Spruit, H. C. 2006, Proceedings of the VI Microquasar Workshop: Microquasars and Beyond. September 18-22, Como, Italy, 44.1

Spruit, H. C., \& Usdensky, D. A. 2005, ApJ, 629, 960

Tadhunter, C. N., Morganti, R., Alighieri, S. S., et al. 1993, MNRAS, 263, 999 (T93)

Tout, C. A., \& Pringgle, J. E. 1996, MNRAS, 281, 219

Urry, C. M., \& Padovani, P. 1995, PASP, 107, 803

Wang, D. X., Xiao, K., \& Lei, W. H. 2002, MNRAS, 335, 665

Wilkes, B. J. 1986, MNRAS, 218, 331 (W86) 